\title{
Erratum to: Cleanup of atrazine-contaminated soils: ecotoxicological study on the efficacy of a bioremediation tool with Pseudomonas sp. ADP
}

\author{
Sónia Chelinho • Matilde Moreira-Santos • Débora Lima • Cátia Silva • Paula Viana \\ Sandra André • Isabel Lopes • Rui Ribeiro • Arsénio M. Fialho • Cristina A. Viegas • \\ José P. Sousa
}

Published online: 9 April 2010

(C) Springer-Verlag 2010

Erratum to: J Soils Sediments

DOI 10.1007/s11368-009-0145-2

Unfortunately Table 4 contained a mistake.

On the first column, where is "10 (leachates)" should be "10 (eluates)".

The online version of the original article can be at http://dx.doi.org/ 10.1007/s11368-009-0145-2.

\footnotetext{
S. Chelinho $(\bowtie) \cdot$ M. Moreira-Santos $\cdot$ C. Silva $\cdot$ R. Ribeiro J. P. Sousa IMAR-CMA, Department of Zoology, University of Coimbra, Largo Marquês de Pombal, 3004-517 Coimbra, Portugal

e-mail: sonia.chelinho@iav.uc.pt

D. Lima $\cdot$ A. M. Fialho $\cdot$ C. A. Viegas

IBB-Institute for Biotechnology and Bioengineering,

Centre for Biological and Chemical Engineering,

Instituto Superior Técnico,

1049-001 Lisboa, Portugal

P. Viana $\cdot$ S. André

APA-Agência Portuguesa do Ambiente,

2611-865 Amadora, Portugal

I. Lopes

CESAM-Departamento de Biologia, Universidade de Aveiro, Campus Universitário de Santiago,

3810-193 Aveiro, Portugal
} 
Table 4 Mean ( \pm standard deviation; $n=3$ ) 72-h growth rate of Pseudokirchneriella subcapitata and 9-day reproduction of Daphnia magna (in terms of time to release the first brood and number of juveniles per female), on eluates (prepared at days 5 and 10) and leachates (prepared at day 7) from microcosms contaminated with Atrazerba $(0,1,10$, and 20 times the recommended dose- RD) and subsequently sprayed with (B) and without (NB) the bioremediation tool

\begin{tabular}{|c|c|c|c|c|c|}
\hline \multirow[t]{3}{*}{ Treatment period (days) } & \multirow[t]{3}{*}{ Microcosms } & \multirow{2}{*}{\multicolumn{2}{|c|}{$\frac{\text { P. subcapitata }}{\text { Growth rate (/day) }}$}} & \multicolumn{2}{|l|}{ D. magna } \\
\hline & & & & \multirow{2}{*}{$\begin{array}{l}\text { Time to } 1 \text { st brood (days) } \\
\text { Eluates }\end{array}$} & \multirow[t]{2}{*}{ Nr. juveniles/female } \\
\hline & & Eluates & Leachates & & \\
\hline 5 (eluates) & 0 & $1.4 \pm 0.03$ & $1.6 \pm 0.06$ & $8 \pm 0$ & $18 \pm 1$ \\
\hline \multirow[t]{5}{*}{7 (leachates) } & RD-NB & $0.10 \pm 0.03^{\mathrm{a}}$ & $0.29 \pm 0.06^{\mathrm{a}}$ & $9 \pm 0^{\mathrm{a}}$ & $9 \pm 1^{\mathrm{a}}$ \\
\hline & $10 \times \mathrm{RD}-\mathrm{NB}$ & $0.14 \pm 0.01^{\mathrm{a}}$ & $0.21 \pm 0.06^{\mathrm{a}}$ & $14 \pm 2^{\mathrm{a}}$ & $2 \pm 1^{\mathrm{a}}$ \\
\hline & $10 \times \mathrm{RD}-\mathrm{B}$ & $1.5 \pm 0.03^{\mathrm{b}}$ & $1.4 \pm 0.28^{\mathrm{b}}$ & $8 \pm 0^{\mathrm{b}}$ & $12 \pm 4^{\mathrm{b}}$ \\
\hline & $20 \times \mathrm{RD}-\mathrm{NB}$ & $0.12 \pm 0.05^{\mathrm{a}}$ & $0.13 \pm 0.07^{\mathrm{a}}$ & $11 \pm 4^{\mathrm{a}}$ & $7 \pm 7^{\mathrm{a}}$ \\
\hline & $20 \times \mathrm{RD}-\mathrm{B}$ & $0.23 \pm 0.16$ & $1.1 \pm 0.40^{\mathrm{b}}$ & $14 \pm 4$ & $3 \pm 3$ \\
\hline \multirow[t]{3}{*}{10 (eluates) } & 0 & $0.96 \pm 0.35$ & $\mathrm{nt}$ & $11 \pm 4$ & $9 \pm 9$ \\
\hline & $20 \times \mathrm{RD}-\mathrm{NB}$ & $0.18 \pm 0.04$ & $\mathrm{nt}$ & $15 \pm 1$ & $3 \pm 3$ \\
\hline & $20 \times \mathrm{RD}-\mathrm{B}$ & $0.96 \pm 0.26^{\mathrm{b}}$ & $\mathrm{nt}$ & $9 \pm 1^{b}$ & $10 \pm 3^{\mathrm{b}}$ \\
\hline
\end{tabular}

$n t$ denotes treatment not tested

${ }^{\mathrm{a}}$ Mean statistically different from control

${ }^{\mathrm{b}}$ Mean statistically different from the NB treatment within the same Atrazerba dose 